\title{
Inhibition of high glucose-induced apoptosis by uncoupling protein 2 in human umbilical vein endothelial cells
}

\author{
YING HE, NING WANG, YINCHEN SHEN, ZHI ZHENG and XUN XU \\ Division of Opthalmology, Shanghai First People's Hospital, \\ Shanghai Jiao Tong University School of Medicine, Shanghai 200080, P.R. China
}

Received October 30, 2013; Accepted February 21, 2014

DOI: $10.3892 /$ ijmm.2014.1676

\begin{abstract}
Studies have shown that an overproduction of mitochondrial reactive oxygen species (ROS) is an initiating cause in the pathogenesis of diabetic complications. However, uncoupling protein 2 (UCP2) can protect retinal vascular endothelial cells from damage by inhibiting the overproduction of mitochondrial ROS, although the protective mechanism involved is not completely clear. This study aimed to assess the effect and mechanism of UCP2 on the apoptosis of human umbilical vein endothelial cells (HUVECs). HUVECs were cultured in normal glucose (NG, $5.5 \mathrm{mmol} / \mathrm{l})$ or high glucose ( $\mathrm{HG}, 30 \mathrm{mmol} / \mathrm{l})$ medium in the presence or absence of $\mathrm{UCP} 2^{+/+}$lentiviral transfection. Lentivirus-mediated UCP2 overexpression inhibited the apoptosis of HUVECs induced by HG. Treatment with HG resulted in the upregulation of caspase-3 and cytochrome $c$ and the downregulation of $\mathrm{Bcl}-2$ in vitro. Furthermore, compared with the NG group, the rate of apoptosis was significantly increased in the $\mathrm{HG}$ group. On day two post-infection, NG cells showed significantly greater HUVEC cell proliferation than HG cells. Notably, UCP2 overexpression inhibited these processes. Taken together, these results suggest that UCP2 promotes cell proliferation and inhibits HG-induced apoptosis in HUVECs via the Bcl-2 upand downregulation of caspase- 3 and cytochrome $c$ in vitro. This may provide experimental evidence for the application of UCP2 as a new protective factor for diabetic complications, such as diabetic retinopathy.
\end{abstract}

\section{Introduction}

Diabetic retinopathy (DR) is a major cause of blindness in developed countries, and oxidative stress caused by hyperglycemia is one of the most common causes of diabetic microangiopathy (1). Currently, the specific cause of diabetic

Correspondence to: Dr Xun Xu, Division of Ophthalmology, Shanghai First Hospital of Shanghai Jiao Tong University School of Medicine, 100 Haining Road, Shanghai 200080, P.R. China

Email: drxuxun@sjtu.edu.cn

Key words: uncoupling protein 2, high glucose, apoptosis, human umbilical vein endothelial cell microangiopathy is not completely understood. Recently, a unifying hypothesis has been suggested whereby the production of mitochondrial reactive oxygen species (ROS) in response to chronic hyperglycemia may be the key initiator of four pathogenic pathways: the polyol pathway, increased formation of advanced glycation end-products, activation of protein kinase $\mathrm{C}$, and the hexosamine pathway (1-3). These studies have emphasized the important role of increased mitochondrion ROS production in diabetes complications, including retinopathy. Therefore, mitochondrial ROS may serve as an important target for DR treatment. We previously found (4) that uncoupling protein 2 (UCP2) has a negative regulatory role in ROS generation; however, the exact mechanism remains to be determined.

UCPs are mitochondrial transporters present in the inner membrane of mitochondria. They belong to the family of anion mitochondrial carriers. Five different UCPs have already been identified: UCP1-4 and UCP5, or brain mitochondrial carrier protein 1 (BMCP1). These proteins are expressed in different tissues and play different roles in cellular metabolism. Brain-specific UCP4 and UCP5 have been suggested to play a role in apoptosis in the brain $(5,6)$. Immunocytochemistry of UCP expression in endothelial cells and pericytes showed that UCP1 and UCP2 expression was positive in both types of cells, while UCP3 was negatively expressed (7). UCP2 was initially described by Fleury et al (8). It shares $59 \%$ identity to UCP1 and is expressed most abundantly in mitochondria from the spleen, certain regions of the brain, the pancreas, lungs, stomach, intestine, white adipose tissue, thymocytes, kidney, cardiomyocytes, macrophages and mast cells, but not in muscle, heart, liver, or brown adipose tissue (8-18). Expression of UCP 2 has been shown to result in a decrease in mitochondrial superoxide (19-24). UCP2 has been shown to have a protective effect in the brain, preventing acute damage produced by ischemia (25-28); in the circulatory system, preventing atherosclerotic plaques (29); and in cardiomyocytes, where its overexpression is anti-apoptotic (30). Findings of previous studies have shown that UCP2 plays a role in macrophages and pancreatic $\beta$ cells by affecting the killing capacity and glucose-induced insulin secretion, respectively $(13,19,22,31,32)$.

Considering that the overexpression of UCP2 can inhibit vascular damage in DR and induce pathological changes associated with vascular injury and nerve-tissue degeneration, we aimed to determine whether UCP2 was able to reduce 
high glucose-induced endothelial cell apoptosis and protect blood vessels from damage. In this study, we investigated whether UCP2 inhibits high glucose-induced apoptosis of human umbilical vein endothelial cells (HUVECs) to provide experimental evidence for the application of UCP2 as a new protective factor of DR.

\section{Materials and methods}

Construction and identification of the UCP2 expression plasmid. The primers targeting the human UCP2 gene were synthesized based on a cDNA library (GenePharma, Shanghai, China), and the sequences were as follows: UCP2 forward, 5'-GGAGATACCAAAGCACCGTCAATG-3' and reverse, 5'-AGCACAGTTGACAATGGCATTACG-3'; hACTB forward, 5'-CCCTGGCACCCAGCAC-3' and reverse, 5'-GCC GATCCACACGGAGTAC-3'.

Each of these fragments was amplified, digested with AgeI and then ligated into the pGC-FU-3FLAG vector for $15 \mathrm{~min}$ at 23 and $42^{\circ} \mathrm{C}$. The recombinant was transformed into competent Escherichia coli cells treated with calcium chloride and incubated for $16 \mathrm{~h}$ at $37^{\circ} \mathrm{C}$.

PCR conditions were as follows: $94^{\circ} \mathrm{C}$ for $5 \mathrm{~min}, 30$ cycles at $94^{\circ} \mathrm{C}$ for $30 \mathrm{sec}, 55^{\circ} \mathrm{C}$ for $30 \mathrm{sec}, 72^{\circ} \mathrm{C}$ for $2 \mathrm{~min}$ and then $72^{\circ} \mathrm{C}$ for $10 \mathrm{~min}$. Positive clones, as confirmed by PCR, were chosen for sequencing. The plasmids with the correct sequence were transfected into $293 \mathrm{~T}$ cells, and expression of the protein was observed under a fluorescence microscope (Nikon, Tokyo, Japan). Western blotting was performed to detect the expression of the protein. The virus titer was determined by quantitative PCR (qPCR).

Cell culture and lentivirus transduction. HUVECs were obtained from the ScienCell Research Laboratories (San Diego, CA, USA). The cells were cultured in Endothelial Cell Medium (ECM; ScienCell Research Laboratories, Carlsbad, CA, USA) with $5 \%(\mathrm{v} / \mathrm{v})$ fetal bovine serum (FBS) at $37^{\circ} \mathrm{C}$ in $5 \%(\mathrm{v} / \mathrm{v})$ $\mathrm{CO}_{2}$ and $95 \%$ humidity. When they reached confluence, the cells were maintained in $1 \%(\mathrm{v} / \mathrm{v})$ fetal calf serum and exposed to normal glucose (NG, $5.5 \mathrm{mmol} / \mathrm{l})$ or high glucose $(\mathrm{HG}$, $30 \mathrm{mmol} / \mathrm{l}$ ) for 3-7 days, during which the medium was changed every 2 days. When the HUVECs were $\sim 50 \%$ confluent in fresh serum-free medium, they were transiently transfected with control lentivirus or UCP2-overexpression lentivirus at a multiplicity of infection (MOI) of 100. The cells were cultured in ECM with 5\% (v/v) FBS after infection for $4 \mathrm{~h}$ and then selected using $200 \mu \mathrm{m} / \mathrm{ml}$ puromycin. The stable overexpressing lines were established when $>90 \%$ of the transfected cells were found to strongly express GFP under a fluorescent microscope. The HUVECs were divided into four groups: $\mathrm{NG}, \mathrm{HG}$, high glucose + the lentiviral-negative vector control group (NC), and high glucose $+\mathrm{UCP} 2^{+/+}$-transfected group (UCP2).

RNA isolation and RT-qPCR. Total RNA was isolated from HUVECs using TRIzol reagent (Invitrogen, Carlsbad, CA, USA) according to the manufacturer's instructions. RNA extract $(2 \mu \mathrm{l})$ was reverse-transcribed into cDNA in a total reaction volume of $20 \mu \mathrm{l}$ using a RevertAid ${ }^{\mathrm{TM}}$ First Strand cDNA Synthesis kit (Fermentas, Burlington, ON, Canada). qPCR was performed using IQ Supermix (Bio-Rad, Hercules, CA, USA), with $20 \mu \mathrm{l}$ reaction mixtures containing $1 \mu \mathrm{l} \mathrm{cDNA}, 8 \mu \mathrm{l}$ sterilized water, $10 \mu \mathrm{l}$ SYBR-Green real-time PCR Master Mix (Takara Bio Inc., Shiga, Japan), and $1 \mu 1$ of primer. The UCP2, cytochrome $c$, caspase-3 and Bcl-2 amplification signals were normalized to glyceraldehyde-3-phosphate dehydrogenase (GAPDH) expression and evaluated using the equation: fold-change $=2^{-\Delta \Delta c t}$. The primer sequences used in this study were: hUCP2 forward, 5'-GGCTGGAGGTGGTCGGAG-3' and reverse, 5'-CAGAAGTGAAGTGGCAAGGGAG-3'; cytochrome $c$ forward 5'-TTTATTATGAAGTGTTCCCAGT GCC-3' and reverse, 5'-CTCCCCAGATGATGCCTTTG-3'; caspase-3 forward, 5'-CAGCCGCCAATAAGAACAAAG-3' and reverse, 5'-CCGCCTCACAATAGCACCC-3'; Bcl-2 forward, 5'-TACCTGAACCGGCACCTG-3' and reverse, 5'-GCCGTACAGTTCCACAAAGG-3'; hGAPDH forward, 5'-GGGTGTGAACCATGAGAAGTATG-3' and reverse, 5'-GATGGCATGGACTGTGGTCAT-3'.

Protein extraction and western blot analysis. HUVECs were washed three times with ice-cold phosphate-buffered saline (PBS, $4^{\circ} \mathrm{C}, \mathrm{pH} 7.4$ ) for $5 \mathrm{~min}$ at room temperature and prepared using a protein extraction kit and a protease inhibitor kit (Pierce, Rockford, IL, USA). The supernatant was collected and the protein content of each lysate was determined using a BCA Protein Assay kit (Tianlai Shengwu Jishu, Tianlai, China) according to the manufacturer's instructions. Equal amounts $(15 \mu \mathrm{l})$ of protein were electrophoresed on a $10 \%(\mathrm{w} / \mathrm{v})$ sodium dodecyl sulfate (SDS) polyacrylamide gel and transferred onto a $0.22 \mu \mathrm{m}$ PVDF membrane (Millipore, Billerica, MA, USA). The primary antibodies used to probe the membranes included anti-UCP2 (1:500; cat. no. ab7973), anti-Bcl-2 (1:500; cat. no. ab7973) (both from Abcam, Cambridge, UK), anti-cytochrome $c$ (1:200; cat no. 1896-1; Epitomics, Burlingame, CA, USA), anti-caspase-3 (1:500; cat. no. ab44976; Abcam) and anti-GAPDH (1:3000; cat. no. KM9002; Sungene Biotech, Tianjin, China). The membranes were washed and incubated with peroxidase-conjugated secondary antibodies (rabbit 1:5,000; rat 1:2,000; Sungene Biotech). Enhanced chemiluminescence western blotting detection reagents (Pierce) were used to detect UCP2, Bcl-2, cytochrome $c$ and caspase-3 protein levels. Experiments were performed in triplicate.

Flow cytometry. According to the manufacturer's instructions, the Annexin-V FITC apoptosis detection kit (Beyotime, Shanghai, China) was used to measure apoptosis in NG, HG, $\mathrm{NC}$ and UCP2 cells. Cells $\left(1 \times 10^{5}\right)$ were plated in a volume of $1 \mathrm{ml}$ into each well of a 6-well plate. After various incubation periods, cells were trypsinized, and the cells and culture medium were collected. Following centrifugation at $1,000 \mathrm{x} \mathrm{g}$ for $10 \mathrm{~min}$, the supernatant was discarded. The cells were resuspended in $1 \mathrm{ml} \mathrm{PBS}$, and transferred to an Eppendorf tube. Annexin-V FITC was added and mixed on ice and left in the dark for $15 \mathrm{~min}$. Cell apoptosis was detected by flow cytometry. Experiments were performed in triplicate.

Immunofluorescence and confocal microscopy. Cells cultured on glass coverslips were fixed for $15 \mathrm{~min}$ at $4^{\circ} \mathrm{C}$ in $\mathrm{PBS}$ containing $4 \%(\mathrm{w} / \mathrm{v})$ paraformaldehyde. The fixative was removed, and cells were either permeabilized with $0.5 \%(\mathrm{v} / \mathrm{v})$ Triton X-100 in PBS for 6-7 min or were not permeabilized. 
Cells were then blocked with $4 \%$ (w/v) bovine serum albumin in PBS for $30 \mathrm{~min}$ at room temperature. The non-permeabilized and permeabilized cells were treated with Bcl-2, cytochrome $c$, caspase-3 monoclonal antibody (1:100, 1:300 and 1:300, respectively) and/or rabbit polyclonal antibody for different organelle markers $(2-5 \mu \mathrm{g} / \mathrm{ml})$ overnight at $4^{\circ} \mathrm{C}$. After removing the unbound primary antibodies and washing them five times with PBS containing 0.5\% (v/v) Triton X-100 (PBST), the cells were incubated with Oregon Green-conjugated (excitation/emission wavelength 496/524 nm) or Rhodamine Red-conjugated (excitation/emission wavelength, 570/590 nm) anti-rabbit secondary antibodies for $40 \mathrm{~min}$ at room temperature. The cells were washed and then stained with $1 \mu \mathrm{g} / \mathrm{ml}$ 4',6-diamidino-2-phenylindole (DAPI) nuclear dye for $10 \mathrm{~min}$ at $37^{\circ} \mathrm{C}$. After the cells were washed five times with PBST, coverslips were mounted onto glass slides and sealed with quenching agent and glycerol. Confocal images were acquired using an Olympus Fluoview 1000 microscope (Olympus, Center Valley, PA, USA). Experiments were performed in triplicate.

Cell viability. We performed the CCK8 assay to monitor cell proliferation. Each group of cells was washed, counted, and seeded at a density of $4 \times 10^{4}$ cells $/ \mathrm{ml}$ in a 96 -well plate. CCK8 solution was added $3 \mathrm{~h}$ before incubation in the various treatments was completed. Cell viability was determined with a spectrophotometer at an absorbance of $450 \mathrm{~nm}$. Experiments were performed in triplicate.

Statistical analysis. SPSS 17.0 was used to analyze experimental data. Experimental findings were presented as the mean \pm standard deviation. One-way ANOVA followed by the Student-Newman-Keuls test was used to compare the effect of treatment on the various parameters. Non-parametric data were analyzed using Chi-square test or the Fisher's exact method. $\mathrm{P}<0.05$ was considered to indicate statistical significance.

\section{Results}

Identification of recombinant plasmid Plenti6.3/V5 DEST. The positive clones were identified after PCR amplification, and the size of the PCR product was $911 \mathrm{bp}$. The detected sequence was identical to the known UCP2 sequence in GenBank (Gene ID NM 003355.2). Western blotting revealed a $32 \mathrm{kDa}$ band in cell extracts, which was in accordance with the expected size of the UCP2-Flag protein $(32 \mathrm{kDa})$. These results indicated that the UCP2 recombinant plasmid was successfully expressed in HUVECs and suggested that these cells were successfully transduced with lentivirus.

Overexpression of UCP2 by stable transfection of Plenti6.3/V5 DEST. Total mRNA and protein extracts were prepared from untransfected controls ( $\mathrm{NG}$ and $\mathrm{HG}$ ), lentiviral-negative vector controls (NC) and UCP2-transfected (UCP2) HUVECs. The UCP2 mRNA and protein expression levels in these cells were determined by RT-qPCR and western blot assays, respectively. RT-qPCR demonstrated that the mRNA levels of UCP2 protein expression in the transfectants containing Plenti6.3/V5 DEST were increased 3-fold when compared with the untreated control HUVECs, which
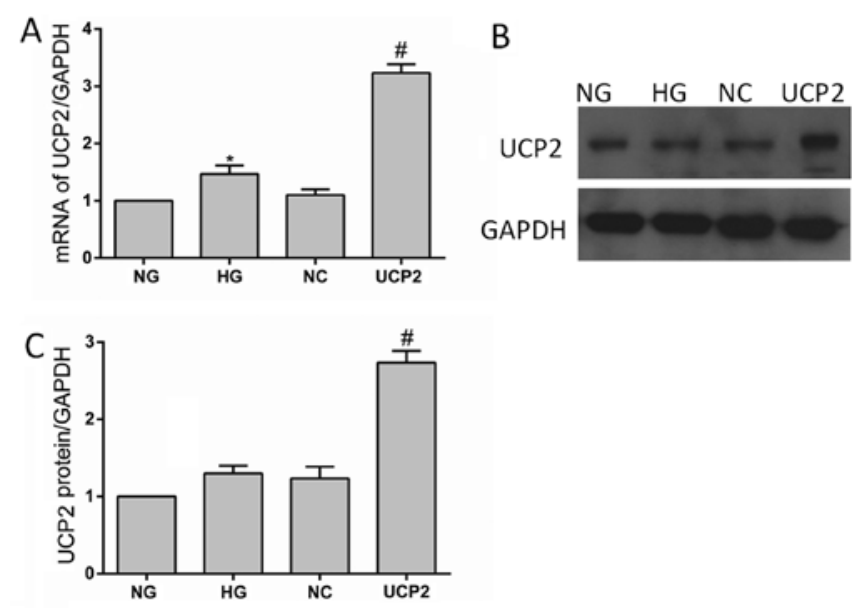

Figure 1. Effect of Plenti6.3/V5 DEST on uncoupling protein 2 (UCP2) expression in human umbilical vein endothelial cells (HUVECs) as determined by RT-qPCR and western blotting. (A) Messenger RNA and (B and C) protein expression of UCP2 in HUVECs transfected with Plenti6.3/V5 DEST were increased when compared with the control groups ( $\mathrm{P}<0.05$ vs. normal glucose group; ${ }^{\text {}} \mathrm{P}<0.05$ vs. high glucose group).

was consistent with the increase of UCP2 protein expression (Fig. 1). There was no significant difference between the cells transfected with the control lentiviral-negative vector and the untransfected cells $(\mathrm{p}>0.05)$. These results indicated that the stable transfection of Plenti6.3/V5 DEST upregulated UCP2 expression in HUVECs.

UCP 2 promoted HUVEC cell proliferation at HG concentrations. The CCK8 assay (Fig. 2) revealed a gradual increase in HUVEC cell proliferation from day one to six in the four cell lines post-infection. On day one post-infection, there was no statistically significant difference in the proliferation of HUVECs among the four cell lines by ANOVA ( $p>0.05)$. On day two post-infection, NG cells showed significantly greater HUVEC cell proliferation than the HG and NC cells $(\mathrm{p}<0.05)$, and UCP2 cells showed significantly greater HUVEC cell proliferation as compared to NC cells $(\mathrm{p}<0.05)$. However, there was no statistically significant difference in HUVEC cell proliferation between the HG and NC cells ( $p>0.05)$. The difference between cell lines over the six successive days was statistically significant by ANOVA.

UCP2 attenuated high glucose-induced apoptosis in HUVECs. With Annexin V FITC labeling, cell apoptosis was analyzed using flow cytometry on day three post-infection. The apoptotic rate in the NG group was $9.31 \%$ (Fig. 3A). This apoptotic rate was significantly different when compared with the HG, NC or UCP2 group $(\mathrm{p}<0.05)$, with the apoptotic rates being $28.73,25.25$ and $13.5 \%$, respectively (Fig. 3A). There was no significant difference in the apoptotic rate between the HG and NC groups (p>0.05) (Fig. 3).

Differing effects of UCP 2 on apoptosis-related protein expression. We investigated whether the overexpression of UCP2 induced by Plenti6.3/V5 DEST affected apoptosis-associated gene expression, such as caspase-3, cytochrome $c$ and Bcl-2. mRNA and protein levels of the three proteins in the NG-, 


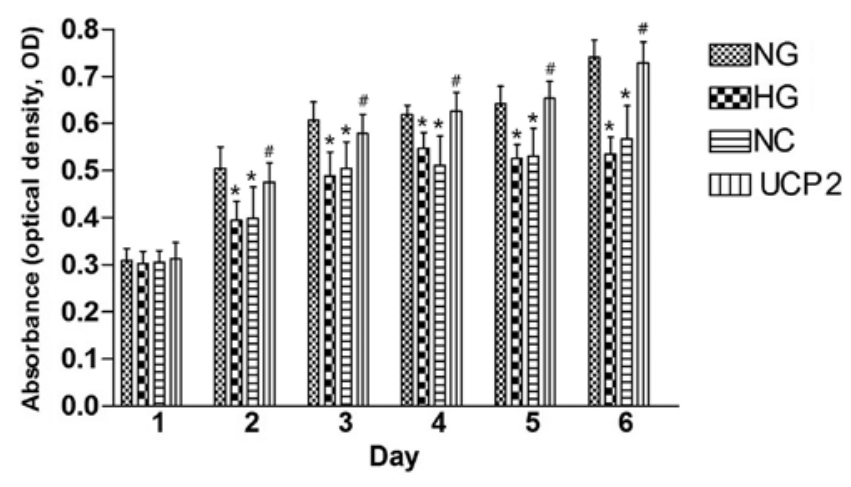

Figure 2. Effect of uncoupling protein 2 (UCP) on the proliferation of human umbilical vein endothelial cells (HUVECs) in the presence of high glucose. The CCK8 assay was used to determine the proliferation of HUVECs. Data are expressed as the mean $\pm \mathrm{SD}$ ( $\mathrm{n}=3$ in each group). ( $\mathrm{P}<0.05$, vs. normal glucose group; ${ }^{\#} \mathrm{P}<0.05$, vs. high glucose group).

HG-, NC- and UCP2-transfected cells were determined by RT-qPCR and western blotting, respectively. The expression trend of the three proteins was found to be consistent with the flow cytometric results. When compared with the NG group, caspase-3 and cytochrome $c$ expression in the HG or $\mathrm{NC}$ group was significantly greater than that in the NG group $(\mathrm{p}<0.05)$, while the anti-apoptotic Bcl-2 expression in the HG or NC group was significantly lower than that in the NG group $(\mathrm{p}<0.05)$. The protein levels of caspase-3, cytochrome $c$ and Bcl-2 showed no significant changes in the HG and NC groups ( $\mathrm{p}>0.05$ ). Caspase -3 and cytochrome $c$ expression in the UCP2 group was significantly lower than that in the $\mathrm{NC}$ group ( $\mathrm{p}<0.05)$, while the anti-apoptotic Bcl-2 expression in the UCP2 group was significantly greater than that in the NC group (p<0.05) (Fig. 4A-D). We also analyzed the levels of the three proteins in the conditioning media by RT-qPCR. The results were consistent with our western blot analysis (Fig. 4E-G).

Localization of apoptosis-related proteins in HUVECs. HUVECs were either labeled against the primary antibodies for caspase-3, cytochrome $c$ or Bcl-2 and nuclei were labeled with blue fluorescent DAPI. Lentivirus-transfected cells were labeled with green fluorescent EGFP. Laser confocal scanning microscopy was employed to observe alterations in the localization of the three proteins. The localized region appeared yellow or orange-yellow. All three proteins were distributed throughout HUVECs, with the fluorescent density in the cytoplasmic area being relatively strong and aggregating in the nuclear periphery. Fluorescence in the nucleus was not well distributed and clustered in aggregates in the plasmosome. When compared with the NG group, caspase-3 and cytochrome $c$ density in the HG or NC group was significantly stronger than that in the NG group, while the anti-apoptotic Bcl-2 density in the $\mathrm{HG}$ or $\mathrm{NC}$ group was significantly weaker than that in the NG group. The protein densities of caspase-3, cytochrome $c$ and Bcl-2 showed no significant changes in the HG and NC groups. Caspase-3 and cytochrome $c$ densities in the UCP2 group were significantly weaker than those in the $\mathrm{NC}$ group $(\mathrm{p}<0.05)$ while the anti-apoptotic Bcl-2 density in the UCP2 group was significantly stronger than that in the
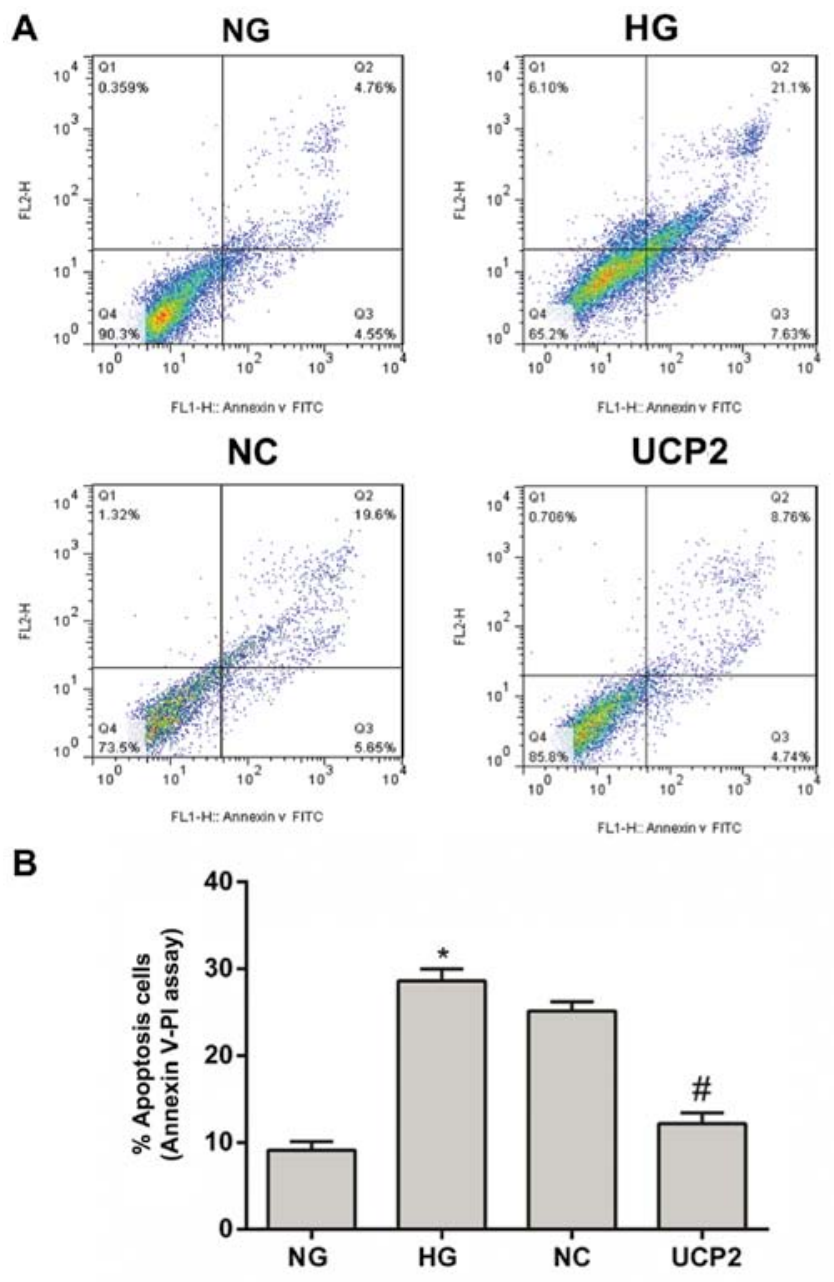

Figure 3. Effect of uncoupling protein 2 (UCP2) on apoptosis of human umbilical vein endothelial cells (HUVECs) induced by high glucose. Apoptosis was analyzed by Annexin V FITC/PI staining at $48 \mathrm{~h}$ after treatment. Data are representative pictures: normal glucose group; high glucose group; lentiviral negative vector control group; UCP2 group.

NC group (Fig. 5). The density trend of the three proteins was found to be consistent with western blotting and RT-qPCR.

\section{Discussion}

In comparison to hUCP1, hUCP2 was found to have a greater effect on mitochondrial membrane potential when expressed in yeast. It was found to have properties consistent with a role in diabetes and obesity. Sayeed et al (33) found that UCP2 silencing in poorly differentiated breast tumor cells rapidly led to the induction of apoptosis and cell differentiation. However, whether UCP2 plays a role in apoptosis induced by HG remains unclear. In this study, we found that UCP2 promoted HUVEC proliferation and attenuated high glucose-induced apoptosis. Beltramo et al (34) found that apoptosis of endothelial cells and pericytes increased in the presence of high levels of glucose after 3 days of culture. In previous studies (35-37) it has been reported that cultured retinal pericytes exposed to high levels of glucose (25-30 mM) for a period of $\geq 7$ days show a higher rate of apoptosis than cells grown at $5.5 \mathrm{mM}$ glucose. Moreover, Cui et al (7) found that cells cultured in HG for 24-25 (23 mM) days and 45-46 (30 $\mathrm{mM})$ days, resulted in an 

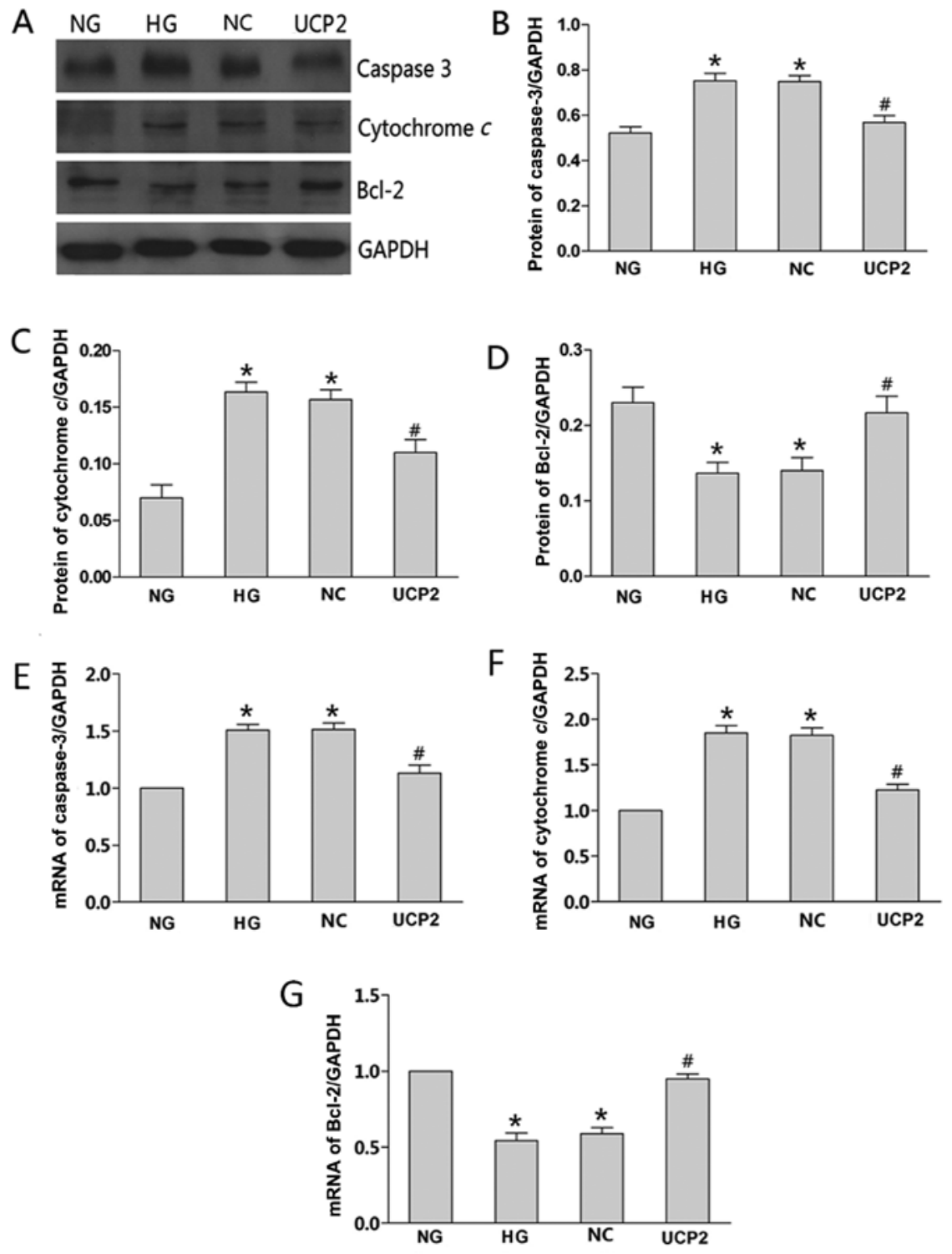

Figure 4. Effect of uncoupling protein 2 (UCP2) on the expression of apoptosis-related proteins as determined by RT-qPCR and western blotting. (A-C) Protein and (E and F) messenger RNA expression of caspase-3 and cytochrome $c$ in the high glucose group or lentiviral-negative vector control group was significantly higher than that in the normal glucose group, while the anti-apoptotic Bcl-2 expression was significantly lower than that in the (D and G) normal glucose group. UCP2 overexpression inhibited these processes. The expression of caspase-3, cytochrome $c$ and Bcl-2 showed no significant changes in the high glucose group and lentiviral-negative vector control group. ( $\mathrm{P}<0.05$ vs. normal glucose group; ${ }^{*} \mathrm{P}<0.05$ vs. high glucose group).

increased rate of apoptosis in pericytes and endothelial cells when compared with cells exposed to low glucose concentrations $(5 \mathrm{mM})$. There were no significant differences between the rates of apoptosis in the 23 and $30 \mathrm{mM}$ glucose groups. Our results were consistent with these studies. Compared with the NG group, the apoptotic rate was significantly increased in the HG and NC groups, while the apoptotic rate in the UCP2 group was significantly decreased. There was no significant difference in the rate of apoptosis between the HG and NC groups. Thus, we concluded that UCP2 attenuates high glucose-induced apoptosis in HUVECs.

Evidence suggests that UCP2 is related to cell proliferation. Elorza et al (38) found that $\mathrm{UCP} 2$ deficiency results in a significant decrease in cell proliferation at the erythropoietin-dependent phase of erythropoiesis. By contrast, Pecqueur et al (39) found that UCP2 $2^{-/-}$cells exhibit enhanced proliferation associated with a metabolic switch from fatty acid oxidation to glucose metabolism. At present, the results are controversial. Therefore, to solve this problem, we tested $\mathrm{UCP}^{+/+}$HUVEC cell proliferation following HG treatment. Our results were consistent with those of Pecqueur et al (39). On day one post-infection, there was no statistically significant difference in the proliferation of HUVECs among the four cell lines. On day two post-infection, NG cells showed significantly greater HUVEC cell proliferation than the HG and NC cells, and UCP2 cells showed significantly greater HUVEC cell proliferation than the NC cells. However, there was no statistically significant difference in HUVEC cell proliferation between the HG and NC cells. Thus, UCP2 promoted HUVEC proliferation.

Apoptosis is a programmed cell death process that serves to remove abnormally proliferative cells, and UCP2 has been previously associated with apoptotic and proliferative activity in poorly differentiated breast tumor cells. However, various 

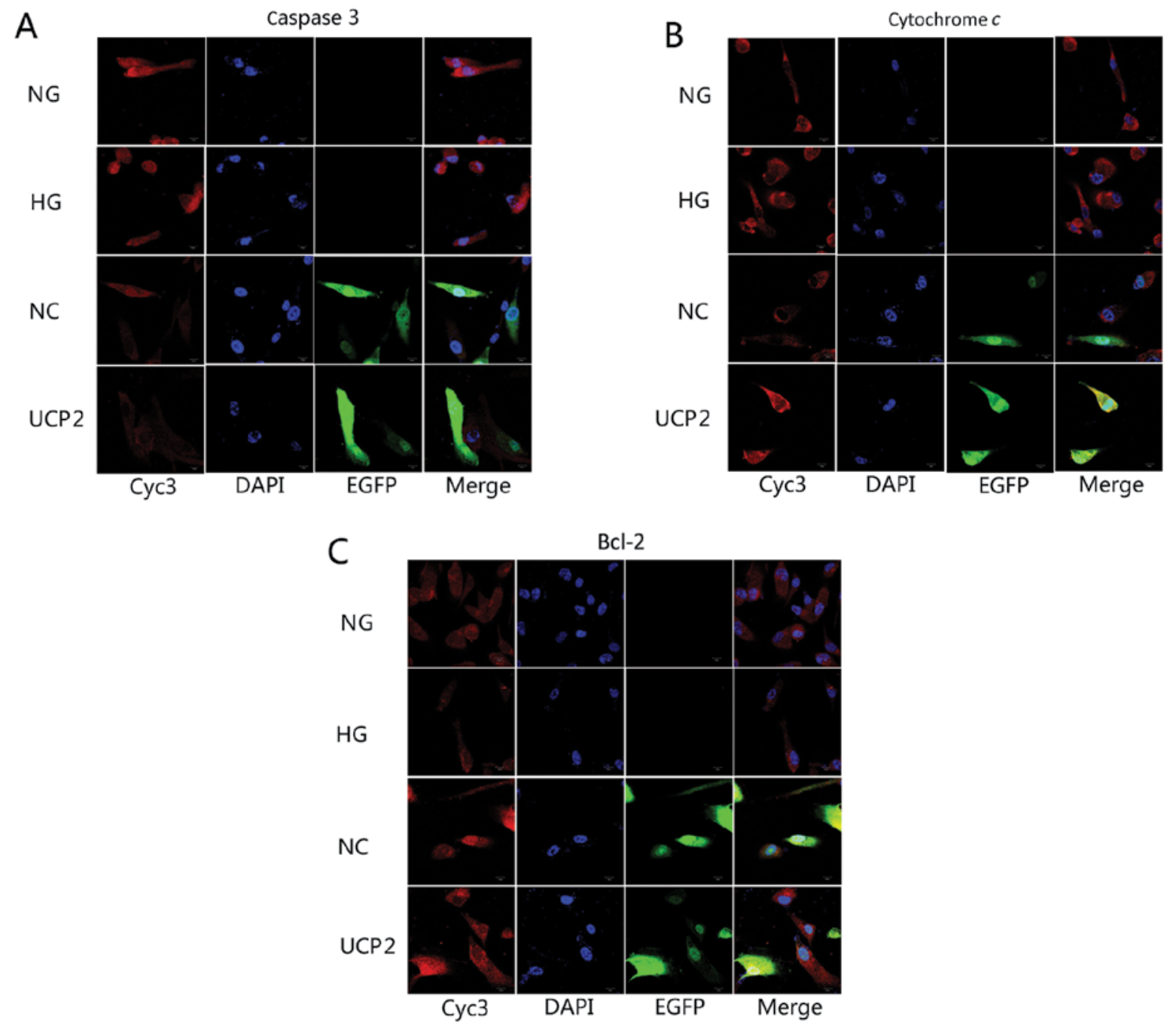

Figure 5. Localization of apoptosis-related proteins in human umbilical vein endothelial cells (HUVECs). The antibodies of caspase-3, cytochrome $c$ and Bcl-2 were labeled with red fluorescent $\mathrm{Cyc} 3$ and the cell nucleus was labeled with blue fluorescent DAPI. Lentivirus-transduced cells were labeled with green fluorescent EGFP. Fluorescence in the nucleus was not well distributed and clusters aggregated in the plasmosome. When compared with the normal glucose group, caspase-3 and cytochrome $c$ density in the high glucose or lentiviral-negative vector control group were significantly stronger than those in the normal glucose group, while the anti-apoptotic Bcl-2 density in the high glucose or lentiviral-negative vector control group was significantly weaker than that in the normal glucose group. The protein densities of caspase-3, cytochrome $c$ and Bcl-2 showed no significant changes in the high glucose and lentiviral-negative vector control group. Caspase-3 and cytochrome $c$ densities in the UCP2 group were significantly weaker than those in the lentiviral-negative vector control group $(\mathrm{P}<0.05)$, while the anti-apoptotic Bcl-2 density in the UCP2 group was significantly stronger than that in the lentiviral-negative vector control group (A-C).

cell types and rat species have been shown to use different mechanisms of UCP2 to influence both apoptotic and proliferative activity. For example, Sayeed et al (33) showed that the negative regulation of UCP2 by TGF $\beta$ signaling rapidly leads to the induction of apoptosis and cell differentiation. In addition, proliferation signaling pathways such as Rb/E2F, c-Myc, and Ras are characterized by their ability to activate nuclear transcription factors located in the cytosol (40). However, Elorza et al (38) showed that UCP2 modulates cell proliferation through the MAPK/ERK pathway during erythropoiesis. Furthermore, Pecqueur et al (39) demonstrated that UCP2 controls proliferation by promoting fatty acid oxidation and limiting glycolysis-derived pyruvate utilization.

To investigate the effect of UCP 2 on cell apoptosis and proliferation, we focused on the expression of the apoptosis-related proteins caspase-3, cytochrome $c$ and $\mathrm{Bcl}-2$, which determine cell fate at the mitochondrial level. Specifically, western blotting and RT-qPCR were used to detect expression levels of the three proteins. Compared with the NG group, caspase-3 and cytochrome $c$ expression in the $\mathrm{HG}$ or NC group was significantly increased, while the anti-apoptotic Bcl-2 expression in the $\mathrm{HG}$ or NC group was significantly decreased. Moreover, protein levels of caspase-3, cytochrome $c$ and Bcl-2 showed no significant changes in the HG and NC groups. Notably, caspase-3 and cytochrome $c$ expression in the UCP2 group was significantly lower than that in the $\mathrm{NC}$ group, while the anti-apoptotic Bcl-2 expression in the UCP2 group was significantly higher than that in the NC group. Therefore, UCP2 promotes cell proliferation and inhibits HG-induced apoptosis in HUVECs via the Bcl-2 up- and downregulation of caspase-3 and cytochrome $c$.

In conclusion, to the best of our knowledge, these are the first results showing that UCP2 promotes cell proliferation and inhibits HG-induced apoptosis in HUVECs via Bcl-2 upand downregulation of caspase- 3 and cytochrome $c$ in vitro. Future efforts should focus on whether these effects are also present in vivo. 


\section{Acknowledgements}

This study was supported by grants from the Research Fund for the National Nature Science Funding of China (nos. 30930097, 81273424 and 81170862), Major National Science and Technology projects during the 12th Five-Year Plan (2011ZX09302-007-02).

\section{References}

1. Forbes JM, Coughlan MT and Cooper ME: Oxidative stress as a major culprit in kidney disease in diabetes. Diabetes 57: 1446-1454, 2008.

2. Brownlee M: Biochemistry and molecular cell biology of diabetic complications. Nature 414: 813-820, 2001.

3. Brownlee M: The pathobiology of diabetic complications: a unifying mechanism. Diabetes 54: 1615-1625, 2005.

4. Zheng Z, Chen H, Ke G, Fan Y, Zou H, et al: Protective effect of perindopril on diabetic retinopathy is associated with decreased vascular endothelial growth factor-to-pigment epitheliumderived factor ratio: involvement of a mitochondria-reactive oxygen species pathway. Diabetes 58: 954-964, 2009.

5. Mattson, MP and Kroemer G: Mitochondria in cell death: novel targets for neuroprotection and cardioprotection. Trends Mol Med 9: 196-205, 2003.

6. Mattson MP and Liu D: Mitochondrial potassium channels and uncoupling proteins in synaptic plasticity and neuronal cell death. Biochem Biophys Res Commun 304: 539-549, 2003.

7. Cui Y, Xu X, Bi H, Wu J, et al: Expression modification of uncoupling proteins and MnSOD in retinal endothelial cells and pericytes induced by high glucose: the role of reactive oxygen species in diabetic retinopathy. Exp Eye Res 83: 807-816, 2001.

8. Fleury C, Neverova M, Collins S, Raimbault S, et al: Uncoupling protein-2: a novel gene linked to obesity and hyperinsulinemia. Nat Genet 15: 269-272. 1997.

9. Pecqueur C, Couplan E, Bouillaud F and Ricquier D: Genetic and physiological analysis of the role of uncoupling proteins in human energy homeostasis. J Mol Med 79: 48-56, 2001.

10. Pecqueur C, Alves-Guerra MC, Gelly C, Levi-Meyrueis C, Couplan $\mathrm{E}$, et al: Uncoupling protein 2 , in vivo distribution, induction upon oxidative stress, and evidence for translational regulation. J Biol Chem 276: 8705-8712, 2001.

11. Couplan E, del Mar Gonzalez-Barroso M, Alves-Guerra MC, Ricquier D, Goubern M and Bouillaud F: No evidence for a basal, retinoic, or superoxide-induced uncoupling activity of the uncoupling protein 2 present in spleen or lung mitochondria. J Biol Chem 277: 26268-26275, 2002.

12. Zhang CY, Baffy G, Perret P, Krauss S, Peroni O, et al: Uncoupling protein-2 negatively regulates insulin secretion and is a major link between obesity, beta cell dysfunction, and type 2 diabetes. Cell 105: 745-755, 2001.

13. Krauss S, Brand MD and Buttgereit F: Signaling takes a breath - new quantitative perspectives on bioenergetics and signal transduction. Immunity 15: 497-502, 2001.

14. Krauss S, Zhang CY, Scorrano L, Dalgaard LT, St-Pierre J, et al: Superoxide-mediated activation of uncoupling protein 2 causes pancreatic beta cell dysfunction. J Clin Invest 112: 1831-1842, 2003.

15. Turner JD, Gaspers LD, Wang G and Thomas AP: Uncoupling protein-2 modulates myocardial excitation-contraction coupling. Circ Res 106: 730-738, 2010.

16. Horvath TL, Warden CH, Hajos M, Lombardi A, Goglia F, et al: Brain uncoupling protein 2: uncoupled neuronal mitochondria predict thermal synapses in homeostatic centers. J Neurosci 19 : 10417-10427, 1999

17. Liu Y, Chen L, Xu X, Vicaut E and Sercombe R: Both ischemic preconditioning and ghrelin administration protect hippocampus from ischemia/reperfusion and upregulate uncoupling protein-2. BMC Physiol 9: 17, 2009.

18. Della-Morte D, Dave KR, DeFazio RA, Bao YC, Raval AP, et al: Resveratrol pretreatment protects rat brain from cerebral ischemic damage via a sirtuin 1-uncoupling protein 2 pathway. Neuroscience 159: 993-1002, 2009.

19. Arsenijevic D, Onuma H, Pecqueur C, Ricquier D, et al: Disruption of the uncoupling protein-2 gene in mice reveals a role in immunity and reactive oxygen species production. Nat Genet 26: 435-439, 2000.
20. Nègre-Salvayre A, Hirtz C, Carrera G, Casteilla L, et al: A role for uncoupling protein-2 as a regulator of mitochondrial hydrogen peroxide generation. FASEB J 11: 809-815, 1997.

21. Li LX, Skorpen F, Egeberg K, Jørgensen IH and Grill V: Uncoupling protein-2 participates in cellular defense against oxidative stress in clonal beta-cells. Biochem Biophys Res Commun 282: 273-277, 2001

22. Bai Y, Onuma H, Bai X, Medvedev AV, Collins S, et al: Persistent nuclear factor-kappa B activation in Ucp2-/- mice leads to enhanced nitric oxide and inflammatory cytokine production. J Biol Chem 280: 19062-19069, 2005.

23. Nishio K, Qiao S and Yamashita $\mathrm{H}$ : Characterization of the differential expression of uncoupling protein 2 and ROS production in differentiated mouse macrophage-cells (Mm1) and the progenitor cells (M1). J Mol Histol 36: 35-44, 2005.

24. Brand MD, Affourtit C, Esteves TC, Parker N, et al: Mitochondrial superoxide: production, biological effects, and activation of uncoupling proteins. Free Radic Biol Med 37: 755-767, 2004

25. Conti B, Sugama S, Lucero J, Bartfai T, et al: Uncoupling protein 2 protects dopaminergic neurons from acute 1,2,3,6-methyl-phenyl-tetrahydropyridine toxicity. J Neurochem 93: 493-501, 2005.

26. Mattiasson G, Shamloo M, Gido G, Wieloch T, et al: Uncoupling protein-2 prevents neuronal death and diminishes brain dysfunction after stroke and brain trauma. Nat Med 9: 1062-1068, 2003.

27. Paradis E, Clavel S, Bouillaud F, Ricquier D and Richard D: Uncoupling protein 2: a novel player in neuroprotection. Trends Mol Med 9: 522-525, 2003

28. Richard D, Clavel S, Huang Q, Sanchis D and Ricquier D: Uncoupling protein 2 in the brain: distribution and function. Biochem Soc Trans 29: 812-817, 2001.

29. Blanc J, Alves-Guerra MC, Esposito B, Rousset S, Mallat Z, et al: Protective role of uncoupling protein 2 in atherosclerosis. Circulation 107: 388-390, 2003.

30. Teshima Y, Akao M, Jones SP and Marbán E: Uncoupling protein-2 overexpression inhibits mitochondrial death pathway in cardiomyocytes. Circ Res 93: 192-200, 2003.

31. Polonsky KS and Semenkovich CF: The pancreatic beta cell heats up: UCP2 and insulin secretion in diabetes. Cell 105: 705-707, 2001.

32. Suh YH, Kim SY, Lee HY, Song DK, et al: Overexpression of short heterodimer partner recovers impaired glucose-stimulated insulin secretion of pancreatic beta-cells overexpressing UCP2.J Endocrinol 183: 133-144, 2004.

33. Sayeed A, Meng Z, Luciani G, Dairkee SH et al: Negative regulation of UCP 2 by TGF $\beta$ signaling characterizes low and intermediate-grade primary breast cancer. Cell Death Dis 1: e53, 2010.

34. Beltramo E, Berrone E, Buttiglieri S and Porta M: Thiamine and benfotiamine prevent increased apoptosis in endothelial cells and pericytes cultured in high glucose. Diabetes Metab Res Rev 20: 330-336, 2004

35. Li W, Liu X, He Z, Yanoff M, Jian B and Ye X: Expression of apoptosis regulatory genes by retinal pericytes after rapid glucose reduction. Invest Ophthalmol Vis Sci 39: 1535-1543, 1998.

36. Naruse K, Nakamura J, Hamada Y, Nakayama M, Chava S, Komori T, Kato K, Kasuya Y, Miwa K and Hotta N: Aldose reductase inhibition prevents glucose-induced apoptosis in cultured bovine retinal microvascular pericytes. Exp Eye Res 71: 309-315, 2000

37. Romeo G, Liu WH, Asnaghi V, Kern TS and Lorenzi M: Activation of nuclear factor-kappaB induced by diabetes and high glucose regulates a proapoptotic program in retinal pericytes. Diabetes 51: 2241-2248, 2002.

38. Elorza A, Hyde B, Mikkola HK, Collins S and Shirihai OS: UCP2 modulates cell proliferation through the MAPK/ERK pathway during erythropoiesis and has no effect on heme biosynthesis. J Biol Chem 283: 30461-30470, 2008.

39. Pecqueur C, Bui T, Gelly C, Tompson CB, et al: Uncoupling protein-2 controls proliferation by promoting fatty acid oxidation and limiting glycolysis-derived pyruvate utilization. FASEB J 22: 9-18, 2008

40. Sears RC and Nevins JR: Signaling networks that link cell proliferation and cell fate. J Biol Chem 277: 11617-11620, 2002. 